\begin{tabular}{|l|c|c|}
\hline \multicolumn{3}{|c|}{ DJS Vol. 38 (2017) 53-62 } \\
\hline 1969 & Delta Journal of Science & \\
\hline
\end{tabular}

\title{
On the dynamics of Kirschner tumor-immune model A.Zaghrout ${ }^{1}$, M.M.A.El-Sheikh ${ }^{2}$, A.R. El-Namoury ${ }^{3}$, and A.El-Ashry ${ }^{3}$
}

\author{
${ }^{1}$ Faculty of Science for girls, Department of Mathematics, Al-Azhar University, Egypt. \\ ${ }^{2}$ Faculty of Science, Department of Mathematics, Menoufia University, Egypt.. \\ E-mail:msheikh_1999@yahoo.com \\ ${ }^{3}$ Faculty of Science, Department of Mathematics, Tanta University, Egypt.. \\ E-mail:asmaa_el_ashry@yahoo.com
}

\begin{abstract}
A tumor-immune model of Kirschner type is considered. The boundedness of solutions are discussed. Criteria for existence and the stability of equiliria are established. Using similar technique to that we used before in the literature, we study the existence of Hopf-Andronov-Poincaré bifurcation. Using Liapunov function sufficient conditions are guaranteed the existence of a unique periodic asymptotically stable solution for the system are established. Numerical simulations are given to illustrate the results.
\end{abstract}

Key words: Kirschner model, Equilibrium points, Global stability, Hopf bifurcation.

\section{1-Introduction:}

Cancer still considered as one of major causes of death world wide. Cancer starts when unbounded growth of normal cells in the body happens fast. It can also occur when cells lose their ability to die. There are many known causes of cancers that have been documented to date including exposure to chemicals, drinking excess alcohol, excessive sunlight exposure, and genetic differences [10]. The most common cause of cancerrelated death is lung cancer. However, the cause of many cancers still remains unknown. The kind of cancers differs from country to another for example, in Japan, there are many cases of stomach cancer, but this is not the case in other countries (see [12]). In 1920's Lotka and Volterra introduced the idea of using the qualitative theory of ordinary differential equations in mathematical biology, population models, and tumor-immune dynamics ( for a good summry of this subject see [1], and [9]). In 1998 Kirschner and Panetta [7] improved the above works and introduced a 3-dimentinal model. They discussed stability analysis and bifurcation theory to classify behavior of equiliriaof the system. In 2009
Kirschner et al [8] established sufficient conditions that guarantee asymptotic convergence of concentrations of tumor cells using quasi-Liapunov functions technique. In 2012, Tsygvintsev et al [13], derived sufficient conditions for the global stability of the cancer-free equilibrium point.

In this paper we discuss Kirschner and Panetta model, analytically and numerically in a fashion like the work of El-Owaidy and El-Sheikh [5], El-Sheikh and Mahrouf [2] and [3], Zaghrout and El-Sheikh [17] and El-Sheikh et al [4].The model in this paper can be summarized briefly as follows, tumor cells are tracked as a continuous variable as they are large and generally homogeneous; they are defined as $y(t)$. Immune cells are those cells that have been stimulated and are ready to respond to the foreign matter (known as effector cells); they are defined as $x(t)$ and assumed also to be large in number. Finally, effector molecules are represented by $z(t)$.These are self-stimulating proteins for effector cells which produce them. The equations that describe the 
interactions of these state variables are given by the following mathematical system (see[7]):

$$
\begin{aligned}
& \frac{d x}{d t}=c y-\mu_{2} x+\frac{p_{1} x z}{g_{1}+z}+s_{1} \\
& \frac{d y}{d t}=\mathrm{r}_{2} y(1-\mathrm{b} y)-\frac{\alpha x y}{g_{2}+y} \\
& \frac{d z}{d t}=\frac{p_{2} x y}{g_{3}+y}-\mu_{3} z+s_{2} .
\end{aligned}
$$

In equation (1a), the first term represents stimulation by the tumor to generate effector immune cells. The parameter $\mathrm{c}$ is known as the antigenicity or strength of this characteristic. The second term in (1a) represents natural death and the third is the proliferative enhancement effect of IL-2. $\mathrm{s}_{1}$ represents a treatment term where by a physician administers effector cells that have been taken from a patient, stimulated to a large degree, and then subsequently infused back into the patient. In equation (1b), the first term is a logistic growth term for tumor growth, and the second is a clearance term by the effector cells. In equation (1c), IL-2 is produced by effector cells (in a MichaelisMenton fashion, i.e. dose response) and decays via a known half-life (third term). The second term, $\mathrm{s}_{2}$ is a treatment term that represents administration of IL-2 (manufactured) by a physician to a patient, to again stimulate effector cell growth and proliferation. To help with interpretation of the mathematical results, we present a table of parameters for ease of parameter interpretation:

\begin{tabular}{|l|}
\hline $\mathrm{c}$ (antigenicity) \\
\hline $\mathrm{P}_{1}$ (proliferation rate of immune cells) \\
\hline $\mathrm{r}_{2}$ (cancer growth rate) \\
\hline$\mu_{3}$ (half-life of effector molecule) \\
\hline $\mathrm{g}_{1}$ (half sat. for proliferation term) \\
\hline$\mu_{2}$ (death rate of immune cells) \\
\hline $\mathrm{g}_{2}$ (half-sat. for cancer clearance) \\
\hline $\mathrm{b}$ (logistic growth of cancer capacity) \\
\hline $\mathrm{p}_{2}$ (production rate of effector molecule) \\
\hline$\alpha$ (cancer clearance term) \\
\hline $\mathrm{t}$ (time) \\
\hline \multicolumn{1}{|c|}{ Table 1.Parameter Values. } \\
\hline
\end{tabular}

In the present paper we consider the case of immunotherapy with ACI and IL-Z (i.e. $s_{1}>0, s_{2}>0$ ). Our main aim is to discuss analytically the existence, stability, and bifurcation of the steady states and to improve some known results obtained for the Kirschner Panetta system (1). The paper is organized as follows, in Section 2, we discuss the dissipativeness and the existence of equiliria of the system. In Section 3, we study the stability in the neighborhood of each critical points. In Section 4 we give sufficient conditions for the permanence. In section 5 we establish sufficient conditions for existence of a unique asymptotically periodic solution using liapunov function. Our technique used in Sections 4 and 5 depends on those of [15]. Finally, in Section 6, we give numerical simulations to illustrate our theoretical results.

\section{2-Existence and Dissipativeness}

It is clear that the components of the right hand side of the system (1) have continuous partial derivatives on the space

$R_{+}^{3}=\{(x(t), y(t), z(t)): x(t) \geq 0, y(t) \geq 0, z(t) \geq 0\} . \mathrm{T}$ herefore, the solution of the system (1) with non-negative initial conditions, exists and is unique.

Theorem 1 The model system (1) is dissipative.

Proof By (1b), we have

$\frac{d y}{d t} \leq y\left(r_{2}(1-b y)\right.$

i.e.

$y(t) \leq \frac{1}{b+k \exp \left(-r_{2} t\right)}, \quad$ for all $\mathrm{t} \geq 0$, where $\mathrm{k}=\frac{1}{\mathrm{y}(0)}-b$.

Thus

$y(t) \leq \frac{1}{b}$ as $\mathrm{t} \rightarrow \infty$.

This means that $y(t) \leq \frac{1}{b}$, for large $\mathrm{t}>0$. In fact this is consistent with [8].

Now putting

$W=x+y+z$, then

$\frac{d W}{d t} \leq c y-\mu_{2} x+\frac{p_{1} x\left(g_{1}+z\right)}{g_{1}+z}+s_{1}+r_{2} y(1-b y)+\frac{p_{2} x\left(g_{3}+y\right)}{g_{3}+y}-\mu_{3} z+s_{2}$.

But since $\mathrm{y} \leq \frac{1}{b}$, we have

$\frac{d W}{d t}+\theta W \leq s_{1}+s_{2}+\frac{r_{2}}{b}$, where $\theta=\min \left(\mu_{3}, \mu_{2}-\left(p_{1}+p_{2}\right), r_{2}-c\right)$.

So by comparison lemma we obtain,

$W(t) \leq \frac{\left(s_{1}+s_{2}+\frac{r_{2}}{b}\right)}{\theta}-$

$\left(\frac{\left(s_{1}+s_{2}+\frac{r_{2}}{b}\right)}{\theta}-W(\widetilde{T})\right) \exp -\theta(t-\widetilde{T})$, for all $\mathrm{t} \geq \mathrm{T} \geq 0$,

If $\widetilde{\mathrm{T}}=0$, then

$W(t) \leq \frac{\left(s_{1}+s_{2}+\frac{r_{2}}{b}\right)}{\theta}-\frac{\left(s_{1}+s_{2}+\frac{r_{2}}{b}\right)}{\theta}-W(0) \exp -\theta t$

i.e.

$W(t)<\frac{\left(s_{1}+s_{2}+\frac{r_{2}}{b}\right)}{\theta} \quad \forall \mathrm{t} \geq 0$.

So, it follows that all solutions of the system (1) that start 
in $\mathrm{R}_{+}^{3}$ are confined to the region $\Omega$, where

$\Omega=\left\{(x, y, z) \in R_{+}^{3}: W=\frac{\left(s_{1}+s_{2}+\frac{r_{2}}{b}\right)}{\theta}+\varepsilon\right.$ for $\left.\varepsilon>0\right\}(\operatorname{see}[6]$, and $[14])$.

It is clear that the tumor free equilibrium point $E_{1}\left(x_{1}, 0, \frac{s_{2}}{\mu_{3}}\right)$, where $\mathrm{x}_{1}=\frac{s_{1}\left(g_{1} \mu_{3}+s_{2}\right)}{\mu_{2}\left(g_{1} \mu_{3}+s_{2}\right)-p_{1} s_{2}}$, exists if $\mathrm{p}_{1} s_{2}<\mu_{2}\left(\left(g_{1} \mu_{3}+s_{2}\right)\right.$.

Moreover there may exist multiple positive non-trivial steady states, depending on the choice of parameters, $E_{i}=\left(x_{i}, y_{i}, z_{i}\right)$ where $\mathrm{i}$ can range from 1 to 3 . namel $\mathrm{y}$

\section{3- Local Stability and Hopf bifurcation}

Since the Jacobian of the system (1) at any endemic point $(x, y, z)$

$J(x, y, z)=\left[\begin{array}{ccc}\frac{p_{1} z}{g_{1}+z}-\mu_{2} & c & \frac{p_{1} g_{1} x}{\left(g_{1}+z\right)^{2}} \\ -\frac{\alpha y}{g_{2}+y} & r_{2}(1-2 b y)-\frac{\alpha g_{2} x}{\left(g_{2}+y\right)^{2}} & 0 \\ \frac{p_{2} y}{g_{3}+y} & \frac{g_{3} p_{2} x}{\left(g_{3}+y\right)^{2}} & -\mu_{3}\end{array}\right]$.

The characteristic equation at the tumor free equilibrium point $\mathrm{E}_{1}\left(\mathrm{x}_{1}, 0, \frac{\mathrm{s}_{2}}{\mu_{3}}\right)$ is

$\left[\frac{p_{1} s_{2}}{g_{1} \mu_{3}+s_{2}}-\mu_{2}-\lambda\right]\left[r_{2}-\frac{\alpha x_{1}}{g_{2}}-\lambda\right]\left[-\mu_{3}-\lambda\right]$.

The eigenvalues are

$\lambda_{1}=\frac{p_{1} s_{2}}{g_{1} \mu_{3}+s_{2}}-\mu_{2}, \lambda_{2}=r_{2}-\frac{\alpha x_{1}}{g_{2}}, \lambda_{3}=-\mu_{3}$.

Then clearly equilibrium point $\mathrm{E}_{1}$ is asymptotically stable

if $\mathrm{s}_{2}<\frac{\mu_{2} \mu_{1} g_{1}}{p_{1}-\mu_{2}}$, and $\mathrm{s}_{1}>\frac{g_{2} \mu_{2}}{\alpha}\left[\frac{\mu_{2} \mu_{3} g_{1}+s_{2}\left(\mu_{2}-p_{1}\right)}{\mu_{3} g_{1}+s_{2}}\right]$, and unstable otherwise ( This is consistent with [7]).

Now choosing $c$ as a bifurcation parameter for the system (1). Let $c_{c}$ be the value of $c$ at which the characteristic equation on the neighborhood of $E_{i}$, has two pure imaginary roots $\lambda_{1,2}$.

In the following result, we deduce sufficient conditions that guarantee the occurrence of Hopf bifurcation.

Theorem 2 Suppose that the following conditions hold

$\left(A_{1}\right) \frac{p_{1} z_{i}}{g_{1}+z_{i}}<\mu_{2}$

$$
\begin{aligned}
& \left(A_{2}\right) r_{2}\left(1-2 b y_{i}\right)<\frac{\alpha g_{2} x_{i}}{\left(g_{2}+y_{i}\right)^{2}}, \text { and } \\
& \left(A_{3}\right) \frac{p_{1} p_{2} x_{i}\left(g_{2}+y_{i}\right)}{c \alpha}<\left(g_{1}+z_{i}\right)^{2}\left(g_{3}+y_{i}\right) \\
& <\frac{p_{1} z_{i}\left(g_{1}+z_{i}\right)\left(g_{3}+y_{i}\right)+p_{2} p_{1} g_{1} x_{i} y_{i}}{\mu_{3} \mu_{2}},
\end{aligned}
$$

then at $c=c_{c}$ there exists a one parameter family of periodic solutions bifurcating from the critical point $E_{i} \equiv\left(x_{i}, y_{i}, z_{i}\right) \quad$ with period $\mathrm{T}$, where $T \rightarrow T_{\circ}$ as c $\rightarrow$ c and where $T_{\circ}=2 \pi / \omega_{\circ}=2 \pi / \sqrt{\text { trace }^{c}}$. Proof. Since by the assumptions $\left(A_{1}\right)-\left(A_{3}\right)$, there exists at least one real root $\lambda_{3}$ of the cubic equation $\lambda^{3}-($ trace $J) \lambda^{2}+\left(\right.$ trace $\left.J^{c}\right) \lambda-\operatorname{det} J=0$,

where the matrix $J^{c}$ is the first compound of $J$.

Now since

$$
\begin{aligned}
& \text { trace } J=\frac{p_{1} z_{i}}{g_{1}+z_{i}}-\mu_{2}-\mu_{3}+r_{2}\left(1-2 b y_{i}\right)-\frac{\alpha g_{2} x_{i}}{\left(g_{2}+y_{i}\right)^{2}}, \\
& \text { trace }^{c}=\mu_{3}\left[\mu_{2}-\frac{p_{1} z_{i}}{g_{1}+z_{i}}\right]+\left[r_{2}\left(1-2 b y_{i}\right)-\frac{\alpha g_{2} x_{i}}{\left(g_{2}+y_{i}\right)^{2}}\right] \\
& {\left[\frac{p_{1} z_{i}}{g_{1}+z_{i}}-\mu_{2}-\mu_{3}\right]-\frac{p_{2} p_{1} x_{i} y_{i}}{\left(g_{1}+z_{i}\right)^{2}\left(g_{3}+y_{i}\right)}+\frac{c \alpha y_{i}}{g_{2}+y_{i}} \text {, and }} \\
& \operatorname{det} J=-\left[\mu_{3}\left(\frac{p_{1} z_{i}}{g_{1}+z_{i}}-\mu_{2}\right)+\frac{p_{2} p_{1} x_{i} y_{i}}{\left(g_{1}+z_{i}\right)^{2}\left(g_{3}+y_{i}\right)}\right] \\
& {\left[r_{2}\left(1-2 b y_{i}\right)-\frac{\alpha g_{2} x_{i}}{\left(g_{2}+y_{i}\right)^{2}}\right]-\frac{c \alpha y_{i} \mu_{3}}{g_{2}+y_{i}}-} \\
& \frac{\alpha g_{1} g_{3} p_{2} p_{1} x_{i}^{2} y_{i}}{\left(g_{1}+z_{i}\right)^{2}\left(g_{3}+y_{i}\right)^{2}\left(g_{2}+y_{i}\right)} \text {. }
\end{aligned}
$$

So we have the following factorization

$\left(\lambda-\lambda_{3}\right)\left[\lambda^{2}+\left(\lambda_{3}\right.\right.$-trace $\left.J\right) \lambda+\left(\lambda_{3}^{2}-(\right.$ trace $J) \lambda+$ trace $\left.\left.^{c}\right)\right]=0$.

But since by (2), we have

$$
\lambda_{1}+\lambda_{2}+\lambda_{3}=\text { traceJ }
$$

Therefore the remaining roots $\lambda_{1}, \lambda_{2}$ of (2) are of the form

$\lambda_{1,2}=\frac{1}{2}\left\{-\left[\lambda_{3}-\right.\right.$ trace $\left.\left.J\right] \pm \sqrt{\left(\left[\lambda_{3}-\text { trace } J^{2}-4\left(\lambda_{3}^{2}-(\text { trace }) \lambda+\text { trace }^{c}\right)\right.\right.}\right\}$.

Going through as in [5] and [11], we see that at $c=c_{c}$ $\lambda_{3}=$ traceJ, $\lambda_{1}=\overline{\lambda_{2}}$, moreover $\operatorname{Eq}(2)$ can be written as

$$
F_{c}(\text { trace } J)=(\text { trace } J)\left(\text { trace }^{c}\right)-\operatorname{det} J=0 .
$$

It is clear that $\lambda_{3}=$ traceJ $<0$, trace $^{c}>0$ and $\operatorname{det} J<0$, since $\operatorname{det} J<0, c>0$, and $c=c_{c}>0$ is a solution of the critical equation (5). In fact Eq (5) can be represented by the following straight line. 
$a+b c=F_{c}($ traceJ $)=0$, where

$a=-\left[\frac{p_{1} z_{i}}{g_{1}+z_{i}}-\mu_{2}\right]\left[r_{2}\left(1-2 b y_{i}\right)-\frac{\alpha g_{2} x_{i}}{\left(g_{2}+y_{i}\right)^{2}}\right]$

$\left[\frac{p_{1} z_{i}}{g_{1}+z_{i}}-\mu_{2}-2 \mu_{3}+r_{2}\left(1-2 b y_{i}\right)-\frac{\alpha g_{2} x_{i}}{\left(g_{2}+y_{i}\right)^{2}}\right]+$

$\mu_{3}^{2}\left[\frac{p_{1} z_{i}}{g_{1}+z_{i}}-\mu_{2}+r_{2}\left(1-2 b y_{i}\right)-\frac{\alpha g_{2} x_{i}}{\left(g_{2}+y_{i}\right)^{2}}\right]+$

$\frac{\alpha g_{1} g_{3} p_{2} p_{1} x_{i}^{2} y_{i}}{\left(g_{1}+z_{i}\right)^{2}\left(g_{3}+y_{i}\right)^{2}\left(g_{2}+y_{i}\right)}\left[\frac{p_{1} z_{i}}{g_{1}+z_{i}}-\mu_{2}-\mu_{3}+\right.$

$\left.r_{2}\left(1-2 b y_{i}\right)-\frac{\alpha g_{2} x_{i}}{\left(g_{2}+y_{i}\right)^{2}}\right]-\left[\frac{p_{1} z_{i}}{g_{1}+z_{i}}-\mu_{2}\right]$

$\left[\mu_{3}\left(\frac{p_{1} z_{i}}{g_{1}+z_{i}}-\mu_{2}\right)+\frac{g_{1} p_{2} p_{1} x_{i} y_{i}}{\left(g_{1}+z_{i}\right)^{2}\left(g_{3}+y_{i}\right)}\right]$, and

$b=\left[\frac{p_{1} z_{i}}{g_{1}+z_{i}}-\mu_{2}-\mu_{3}+r_{2}\left(1-2 b y_{i}\right)-\frac{\alpha g_{2} x_{i}}{\left(g_{2}+y_{i}\right)^{2}}\right]$.

Conversely, knowing that

det $J<0$ and trace $J<0, c>0$, we can solve equation (5)

for $c_{c}>0$, we then know that trace $_{c_{c}}^{c}>0, \lambda_{3}=$ trace $_{c_{c}}$ and $\lambda_{1}, \lambda_{2}$ are conjugate imaginary.

Since $b>0, \lim _{c \rightarrow \infty} F_{c}($ trace $J)=-\infty$, and $\lim _{c \rightarrow-\infty} F_{c}($ trace $J)=+\infty$,

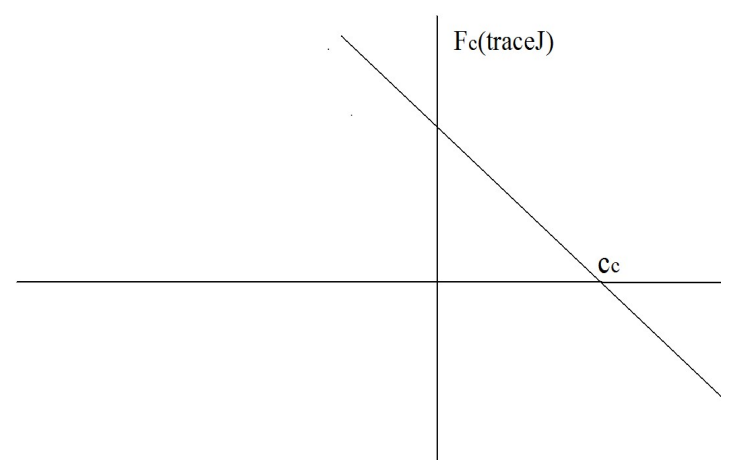

Fig.1. The uniqueness of the bifurcation parameter $c_{c}$

Now, since by (2), $\lambda_{3}=$ trace $J$, and

$$
\begin{gathered}
F_{c_{c}}(\text { trace } J)=\left[\text { trace } J-\lambda_{3}\right]\left[\lambda_{1} \lambda_{2}+(\text { trace } J) \lambda_{3}\right] \\
\operatorname{sgn} F_{c_{c}}(\text { trace } J)=\operatorname{sgn}(\text { trace } J) .
\end{gathered}
$$

Consequent ly if $\mathrm{c}>\mathrm{c}_{\mathrm{c}}$ then $\operatorname{Re} \lambda_{1,2}=\frac{1}{2}\left(\right.$ trace $\left.J-\lambda_{3}\right)<0$, and for $c<c_{c}$, $\operatorname{Re} \lambda_{1,2}>0$ (see Fig.1.)

By the above discussion, we see that as $c$ increased through $c_{c}$, there exists a pair of complex conjugate imaginary eigenvalues $\lambda_{1,2}$ of the Jacobian matrix $J^{c}$.
Since at $c=c_{c}$, then $\lambda_{3}=$ trace $J$, and $\lambda_{1,2}= \pm \sqrt{\text { trace }^{c}}= \pm i \omega_{0}$, where it is clear that $\omega_{\circ}>0$.

Now, Since for $\lambda_{1}=\overline{\lambda_{2}}$, we have

$\operatorname{Re} \lambda_{1,2}=\frac{1}{2}\left(\lambda_{1}+\overline{\lambda_{2}}\right)=0$ at $c=c_{c}$.

So, we have $\operatorname{Re} \lambda_{1,2}>0$ for $c<c_{c}$,

$$
\operatorname{Re} \lambda_{1,2}>0 \text { for } c<c_{c} \text {. }
$$

Moreover

$\left.\frac{d}{d c}\left(\operatorname{Re} \lambda_{1,2}\right)\right|_{c=c_{c}}=-\left.\frac{1}{2}\left(\lambda_{3}-\right.$ trace $)\right|_{c=c_{c}}=\left.\operatorname{Re}\left(\frac{d}{d c} \lambda_{1,2}\right)\right|_{c=c_{c}}<0$.

This completes the proof.

\section{4-Permanence}

We first give the following preliminaries.

Definition 1[15] We say that the system (1) is permanent if there are positive constants $m$ and $M$ such that for each positive solution $\left(\mathrm{x}_{1}(\mathrm{t}), \mathrm{x}_{2}(\mathrm{t}), \mathrm{x}_{3}(\mathrm{t})\right)$ of the system (1) satisfies $m \leq \liminf _{t \rightarrow \infty} x_{i}(t) \leq \lim _{t \rightarrow \infty} \sup x_{i}(t) \leq M$, where $\mathrm{i}=1,2,3$. Definition 2 [15] A solution $X\left(t, t_{0}, \phi\right)$ is called ultimately bounded. If there exists $B>0$ such that for any, $t_{\circ} \geq 0, \phi \in C$, there exists $T=T\left(t_{\circ}, \phi\right)>0$ when $t \geq t_{\circ}+T, \mid X\left(t, t_{\circ}, \phi \mid \leq B\right.$.

\section{Lemma 1[15] If}

$a>0, b>0$, and $\frac{d x}{d t} \geq x(b-a x)$, for $t \geq 0$, and $x(0)>0$,

we have $\liminf _{t \rightarrow \infty} x(t) \geq \frac{b}{a}$, while if

$a>0, b>0$, and $\frac{d x}{d t} \leq x(b-a x)$, for $t \geq 0$, and $x(0)>0$,

we have $\lim _{t \rightarrow \infty} \sup x(t) \geq \frac{b}{a}$.

Now we give the following permanence result.

Theorem 3 Let $\mathrm{M}_{1}, \mathrm{M}_{2}, \mathrm{M}_{3}, \mathrm{~m}_{1}, \mathrm{~m}_{2}$, and $\mathrm{m}_{3}$ be defined by

$M_{1}=\frac{\frac{c}{b}+s_{1}}{\mu_{2}-p_{1}}, M_{2}=\frac{1}{b}, M_{3}=\frac{p_{2} \mu_{1}+s_{1}}{\mu_{3}}, m_{1}=\frac{s_{1}}{\mu_{2}}, m_{2}=\frac{g_{2} r_{2}-\alpha \mu_{1}}{g_{2} r_{2} b}$,

and $m_{3}=\frac{s_{2}}{\mu_{3}}$. Further assume that

$\left(H_{1}\right): p_{1}<\mu_{2}$, and

$\left(\mathrm{H}_{2}\right): \mathrm{g}_{2} \mathrm{r}_{3}>\alpha \mathrm{M}_{1}$, hold, 
Then the system (1) is permanent. This means that there exist positive constants $m_{i}, M_{i}(i=1,2,3)$ which are independent of the solutions of the system (1), such that for any positive solution $\left(\mathrm{x}_{1}(\mathrm{t}), \mathrm{x}_{2}(\mathrm{t}), \mathrm{x}_{3}(\mathrm{t})\right)$ of the system with the initial conditions

$$
x_{i}(0) \geq 0(i=1,2,3),
$$

we have

$$
m_{i} \leq \liminf _{t \rightarrow \infty} x_{i}(t) \leq \lim _{t \rightarrow \infty} \sup x_{i}(t) \leq M_{i} .
$$

Proof. Let $\left(\mathrm{x}_{1}(\mathrm{t}), \mathrm{x}_{2}(\mathrm{t}), \mathrm{x}_{3}(\mathrm{t})\right)$ be any positive solution of the system (1) with the initial value

$\left(x_{1}(0), x_{2}(0), x_{3}(0)\right)$. It follows from the first equation of (1) that

$$
\frac{d x_{1}}{d t}=c x_{2}-\mu_{2} x_{1}+\frac{p_{1} x_{1} x_{3}}{g_{1}+x_{3}}+s_{1} \text {. }
$$

Now by Theorem 1, we have $\frac{d x_{1}}{d t} \leq \frac{c}{b}+s_{1}+\left(p_{1}\left(\frac{g_{1}+x_{1}}{g_{1}+x_{3}}\right)-\mu_{2}\right) x_{1}$.

i.e.

$\frac{d x_{1}}{d t}-\left(p_{1}-\mu_{2}\right) x_{1} \leq \frac{c}{b}+s_{1}$.

According to the theory of differential inequality, we get $x=\frac{\frac{c}{b}+s_{1}}{\mu_{2}-p_{1}}+\left[\frac{\frac{c}{b}+s_{1}}{\mu_{2}-p_{1}}+x(0)\right] \exp \left(p_{1}-\mu_{2}\right) t$.

Further since $p_{1}<\mu_{2}$, then

$$
\limsup _{t \rightarrow \infty} x_{1}(t) \leq \frac{\frac{c}{b}+s_{1}}{\mu_{2}-p_{1}}=M_{1}
$$

Thus for any positive constant $\varepsilon>0$, it follows from (6) that there exists a $T_{1}>0$ such that for all $t>T_{1}$, we have

$$
x_{1} \leq M_{1}+\varepsilon .
$$

Similarly by the second equation of the system , we have by Lemma 1,

$$
\lim _{t \rightarrow \infty} \sup x_{2}(t) \leq \frac{1}{b}=M_{2} .
$$

Consequently for $\varepsilon>0$, it follows that there exists a $T_{2}>0$ such that for all $t>T_{2}$,we get

$$
x_{2} \leq M_{2}+\varepsilon
$$

Similarly from the third equation, we have

$$
x_{2} \leq M_{3}+\varepsilon
$$

But since from (1.a)

$$
\begin{aligned}
\frac{d x_{1}}{d t} & =c x_{2}-\mu_{2} x_{1}+\frac{p_{1} x_{1} x_{3}}{g_{1}+x_{3}}+s_{1} \\
& \geq s_{1}-\mu_{2} x_{1} .
\end{aligned}
$$

It follows that

$$
\liminf _{t \rightarrow \infty} x_{1}(t)=\frac{s_{1}}{\mu_{2}}=m_{1} .
$$

Similarly, from (1.b) and (1.c), we can easily show that

$$
\liminf _{t \rightarrow \infty} x_{2}(t)=\frac{g_{2} r_{2}-\alpha M_{1}}{g_{2} r_{2} b}=m_{2} \text {. }
$$

and

$$
\liminf _{t \rightarrow \infty} x_{3}(t)=\frac{s_{2}}{\mu_{3}}=m_{3} .
$$

Thus the system (1) is permanent.

\section{5-Existence and Uniqueness of Asymptotically Periodic Solution}

Following [15] we consider the asymptotically periodic system as follows,

$$
\frac{d x}{d t}=f\left(t, x_{t}\right)
$$

where $f \in C\left([-r, 0], R^{n}\right)$ and for any $x_{t} \in C$. Define $x_{i}(\theta)=x(t+\theta), \theta \in[-r, 0]$. For any $x=\left(x_{1}, x_{2}, \ldots x_{n}\right) \in R_{n}$ we define $|\mathrm{x}|=\sum_{i=1}^{n}\left|x_{i}\right|$, from sec 4 , it is easy to see that there exists $H>0$, such that $|x| \leq n M_{i}<H$. For any $\phi \in C$, define $\|\phi\|=\sup _{-r \leq \theta \leq 0}|\phi(\theta)|$. Let $C_{H}=\{\phi \in C,\|\phi\|<H\}$, and $S_{H}=\left\{x \in R^{n},|x|<H\right\}$. In this section we use the same technique [15] to discuss the existence and uniqueness of asymptotically periodic solution of system (14), we consider the adjoint system

$$
\begin{aligned}
& \frac{d x}{d t}=f\left(t, x_{t}\right) \\
& \frac{d y}{d t}=f\left(t, y_{t}\right)
\end{aligned}
$$

The following lemma is needed

Lemma 2 (Yuan $[15,16]$ ) Let $V \in\left(R_{+} \times S_{H} \times S_{H}, R_{+}\right.$) satisfy

(i) $a(|x-y|) \leq V(t, x, y) \leq b(\mid x-y), \mid$ where $a(r)$ and

$b(r)$ are are continuously positively increasing functions;

(ii) $\left|V\left(t, x_{1}, y_{1}\right)-V\left(t, x_{2}, y_{2}\right)\right| \leq l\left(\left|x_{1}-x_{2}\right|+\left|y_{1}-y_{2}\right|\right)$, where $l$ is a constant and satisfies $l>0$;

(iii) there exists continuous non-increasing function $P(s)$, such that for $s>0, P(s)>s$, and as $P(V(t, \phi(0), \phi(0))>(V(t+\theta, \phi(\theta), \phi(\theta)), \theta \in[-r, 0]$, it follows that $V_{(16)}^{\prime}(t, \phi(0), \phi(0)) \leq-\delta V(t, \phi(0), \phi(0))$, 
where $\delta$ is a constant and satisfies $\delta>0$. Furthermore, the system (15) has a solution $\zeta(t)$ for $t>t_{\circ}$ and satisfies $\|\zeta(t)\| \leq H$. Then system (14) has a unique asymptotically periodic solution, which is uniformly asymptotically stable.

Theorem 4 Let $\theta_{1}, \theta_{2}, \theta_{3}$ and $\delta$ are defined by

$$
\begin{aligned}
& \theta_{1}=M_{1}\left[\frac{c M_{2}}{M_{1}^{2}}+\frac{s_{1}}{M_{1}^{2}}+\frac{\alpha g_{2}}{\left(g_{2}+M_{2}\right)^{2}}-\frac{p_{2} M_{2}\left(1+g_{3}\right)}{m_{3}\left(g_{3}+m_{2}\right)^{2}}\right] \\
& \theta_{2}=M_{2}\left[r_{2} b-\frac{c}{m_{1}}-\frac{p_{2} M_{1} g_{3}}{m_{3}\left(g_{3}+m_{2}\right)^{2}}\right], \text { and } \\
& \theta_{3}=M_{3}\left[\frac{s_{2}}{M_{3}^{2}}-\frac{p_{1} g_{1}}{\left(g_{1}+m_{3}\right)^{2}}+\frac{p_{1} m_{1} m_{2}\left(m_{2}+g_{3}\right)}{M_{3}^{2}\left(g_{1}+M_{2}\right)^{2}}\right] \\
& \delta=\min \left\{\theta_{1}, \theta_{2}, \theta_{3}\right\}
\end{aligned}
$$$$
\text { respectively. In addition to the conditions }\left(H_{1}\right) \text { and }
$$
$\left(\mathrm{H}_{2}\right)$, we assume further that $\delta>0$, then there exists a unique asymptotically periodic solution of system (1) which is uniformly asymptotically stable.

Proof. By Theorem, we know that the solution of the system (1) is ultimately bounded. Consider the adjoint system of the system (1) as follows $\frac{d x_{1}}{d t}=c x_{2}-\mu_{2} x_{1}+\frac{p_{1} x_{1} x_{3}}{g_{1}+x_{3}}+s$

$$
\frac{d x_{2}}{d t}=\mathrm{r}_{2} x_{2}\left(1-\mathrm{b} x_{2}\right)-\frac{\alpha x_{1} x_{2}}{g_{2}+x_{2}}
$$$$
\frac{d x_{3}}{d t}=\frac{p_{2} x_{1} x_{2}}{g_{3}+x_{2}}-\mu_{3} x_{3}+s_{2}
$$

$\frac{d u_{1}}{d t}=c u_{2}-\mu_{2} u_{1}+\frac{p_{1} u_{1} u_{3}}{g_{1}+u_{3}}+s_{1}$

$\frac{d u_{2}}{d t}=\mathrm{r}_{2} u_{2}\left(1-\mathrm{b} u_{2}\right)-\frac{\alpha u_{1} u_{2}}{g_{2}+u_{2}}$

$\frac{d u_{3}}{d t}=\frac{p_{2} u_{1} u_{2}}{g_{3}+u_{2}}-\mu_{3} u_{3}+s_{2}$.

For

$X(t)=\left(x_{1}(t), x_{2}(t), x_{3}(t)\right)$ and $U(t)=\left(u_{1}(t), u_{2}(t), u_{3}(t)\right)$ are the solutions of system (20) in $\Omega \times \Omega$. Let $x_{i}^{*}(t)=\ln x_{i}(t), u_{i}^{*}=\ln u_{i}(t), i=1,2,3$.

Consider a Liapunov functional in the form

$$
V(t)=\sum_{i=1}^{3}\left|x_{i}^{*}(t)-u_{i}^{*}(t)\right|
$$

By taking $a(r)=b(r)=\sum_{i=1}^{n}\left|x_{i}^{*}(t)-u_{i}^{*}(t)\right|$ and using the inequality ||$a|-| b|| \leq|a-b|$ the proof of condition $(i)$, and (ii) of Lemma 2 be as [15]. Now to prove (iii) of Lemma 2. It follows from (21) that
$D^{+} V(t)=\sum_{i=1}^{n}\left(\frac{x_{i}(t)}{x_{i}(t)}-\frac{u_{i}(t)}{u_{i}(t)}\right) \times \operatorname{sign}\left(x_{i}(t)-u_{i}(t)\right)$,

then we have

$$
\begin{aligned}
& D^{+} V(t) \leq c \frac{x_{2}}{x_{1}}-\mu_{2}+\frac{p_{1} x_{3}}{g_{1}+x_{3}}+\frac{s_{1}}{x_{1}}-c \frac{u_{2}}{u_{1}}+\mu_{2}- \\
& \frac{p_{1} u_{3}}{g_{1}+u_{3}}-\frac{s_{1}}{u_{1}}+r_{2}-r_{2} b x_{2}-\frac{\alpha x_{1}}{g_{2}+x_{2}}-r_{2}+r_{2} b u_{2}-\frac{\alpha u_{1}}{g_{2}+u_{2}} \\
& +\frac{p_{2} x_{1} x_{2}}{x_{3}\left(g_{3}+x_{2}\right)}-\mu_{3}+\frac{s_{2}}{x_{3}}-\frac{p_{2} u_{1} u_{2}}{u_{3}\left(g_{3}+u_{2}\right)}+\mu_{3}-\frac{s_{2}}{u_{3}} .
\end{aligned}
$$

Furthermore,

$$
\begin{aligned}
& D^{+} V(t) \leq\left|x_{1}-u_{1}\right|\left\{\frac{-c x_{2}}{u_{1} x_{1}}-\frac{s_{1}}{u_{1} x_{1}}-\frac{\alpha g_{2}}{\left(g_{2}+x_{2}\right)\left(g_{2}+x_{2}\right)}+\right. \\
& \left.\frac{p_{2} x_{2} u_{2}}{u_{3}\left(g_{3}+u_{2}\right)\left(g_{3}+x_{2}\right)}+\frac{p_{2} g_{3} u_{2}}{x_{3}\left(g_{3}+u_{2}\right)\left(g_{3}+x_{2}\right)}\right\}+\left|x_{2}-u_{2}\right| \\
& \left\{\frac{c}{u_{1}}-r_{2} b+\frac{p_{2} g_{3} x_{1}}{x_{3}\left(g_{3}+u_{2}\right)\left(g_{3}+x_{2}\right)}\right\}+\left|x_{3}-u_{3}\right|\left\{-\frac{s_{2}}{x_{3} u_{3}}+\right. \\
& \frac{p_{1} g_{1}}{\left(g_{1}+u_{3}\right)\left(g_{1}+x_{3}\right)}-\frac{p_{2} x_{1} x_{2} u_{2}}{x_{3} u_{3}\left(g_{3}+u_{2}\right)\left(g_{3}+x_{2}\right)}- \\
& \left.\frac{p_{2} g_{3} u_{1} u_{2}}{x_{3} u_{3}\left(g_{3}+u_{2}\right)\left(g_{3}+x_{2}\right)}\right\} .
\end{aligned}
$$

Using Lemma 2, we get

$$
\begin{aligned}
& D^{+} V(t) \leq\left|x_{1}-u_{1}\right|\left\{\frac{-c m_{2}}{M_{1}^{2}}-\frac{s_{1}}{M_{1}^{2}}-\frac{\alpha g_{2}}{\left(g_{2}+M_{2}\right)^{2}}+\right. \\
& \left.\frac{p_{2} M_{2}^{2}}{m_{3}\left(g_{3}+m_{2}\right)^{2}}+\frac{p_{2} g_{3} M_{2}}{m_{3}\left(g_{3}+m_{2}\right)^{2}}\right\}+\left|x_{2}-u_{2}\right|\left\{\frac{c}{m_{1}}-\right. \\
& \left.r_{2} b+\frac{p_{2} g_{3} M_{1}}{m_{3}\left(g_{3}+m_{2}\right)^{2}}\right\}+\left|x_{3}-u_{3}\right|\left\{-\frac{s_{2}}{M_{3}^{2}}+\frac{p_{1} g_{1}}{\left(g_{1}+m_{3}\right)^{2}}\right. \\
& \left.-\frac{p_{2} m_{1} m_{2}^{2}}{M_{3}^{2}\left(g_{3}+M_{2}\right)^{2}}-\frac{p_{2} g_{3} m_{1} m_{2}}{M_{3}^{2}\left(g_{3}+M_{2}\right)^{2}}\right\} .
\end{aligned}
$$

Since

$$
\begin{aligned}
\left|x_{i}(t)-u_{i}(t)\right| & =\left|\exp \left(x_{i}^{*}(t)\right)-\exp \left(u_{i}^{*}(t)\right)\right| \\
& =\left|\exp \zeta_{i}(t)\right|\left|x_{i}^{*}(t)-u_{i}^{*}(t)\right|,
\end{aligned}
$$

where $\zeta_{i}(t)$ lies between $x_{i}(t)$ and $u_{i}(t)$ then, we have $m_{i}\left|x_{i}^{*}(t)-u_{i}^{*}(t)\right|<\left|x_{i}(t)-u_{i}(t)\right|<M_{i}\left|x_{i}^{*}(t)-u_{i}^{*}(t)\right|, i=1,2,3$.

Substituting from (25) into (23), we get 


$$
\begin{aligned}
& D^{+} V(t) \leq-\left\{\frac{c m_{2}}{M_{1}^{2}}+\frac{s_{1}}{M_{1}^{2}}+\frac{\alpha g_{2}}{\left(g_{2}+M_{2}\right)^{2}}-\frac{p_{2} M_{2}^{2}}{m_{3}\left(g_{3}+m_{2}\right)^{2}}-\right. \\
& \left.\frac{p_{2} g_{3} M_{2}}{m_{3}\left(g_{3}+m_{2}\right)^{2}}\right\} M_{1}\left|x_{1}^{*}(t)-u_{1}^{*}(t)\right|-\left\{r_{2} b-\frac{c}{m_{1}}\right. \\
& \left.-\frac{p_{2} g_{3} M_{1}}{m_{3}\left(g_{3}+m_{2}\right)^{2}}\right\} M_{2}\left|x_{2}^{*}(t)-u_{2}^{*}(t)\right|-\left\{\frac{s_{2}}{M_{3}^{2}}-\frac{p_{1} g_{1}}{\left(g_{1}+m_{3}\right)^{2}}\right. \\
& \left.+\frac{p_{2} m_{1} m_{2}^{2}}{M_{3}^{2}\left(g_{3}+M_{2}\right)^{2}}+\frac{p_{2} g_{3} m_{1} m_{2}}{M_{3}^{2}\left(g_{3}+M_{2}\right)^{2}}\right\} M_{3}\left|x_{3}^{*}(t)-u_{3}^{*}(t)\right| .
\end{aligned}
$$

This can be written as

$$
\begin{gathered}
D^{+} V(t) \leq-\theta_{1}\left|x_{1}^{*}(t)-u_{1}^{*}(t)\right|-\theta_{2}\left|x_{2}^{*}(t)-u_{2}^{*}(t)\right| \\
-\theta_{3}\left|x_{3}^{*}(t)-u_{3}^{*}(t)\right|,
\end{gathered}
$$

where $\theta_{1}, \theta_{2}$, and $\theta_{3}$ are defined in (16)-(18).

Consider $\delta$ as defined in (19). It follows

$$
D^{+} V(t) \leq-\delta V(t)
$$

Then (iii) of Lemma 2 is fulfilled. Therefore the system

(1) has a unique positive asymptotically periodic solution in the domain $\Omega$, which is uniformly asymptotically stable. This completes the proof.

\section{6- Numerical Results}

In this section, we perform numerical simulations with the help of parameter values taken from experimental data from published literature. Using Fourth order Runge-Kutta method through out matlab programme. For this purpose we consider the following parameter values

$\mathrm{c}=.05, \mu_{2}=.03, \mathrm{p}_{1}=.1245, \mathrm{~g}_{1}=2 \times 10^{7}, \mathrm{r}_{2}=.1, \mathrm{~b}=1 \times 10^{-9}$,

$\alpha=1, \mathrm{~g}_{2}=1 \times 10^{5}, \mathrm{p}_{2}=5, \mathrm{~g}_{3}=1000, \mathrm{~m}_{3}=10, \mathrm{~s}_{1}=30$, and

$\mathrm{s}_{2}=20$ with the initial conditions $x_{\circ}=1000, y_{\circ}=1000$, and $\mathrm{z}_{\circ}=1000$. It clear Fig.2. that the system (1) is a stable spiral .

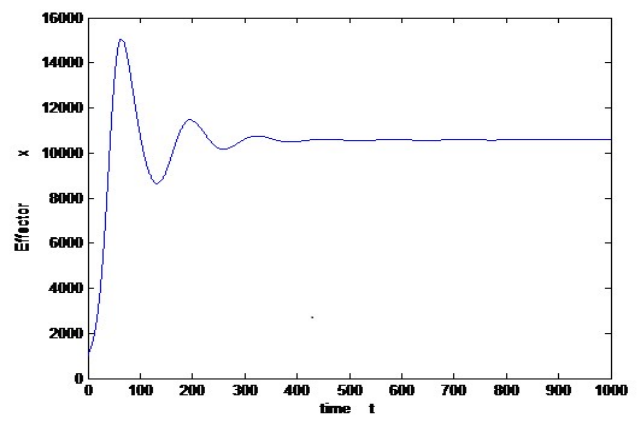

(2-a) The time response of effector cells

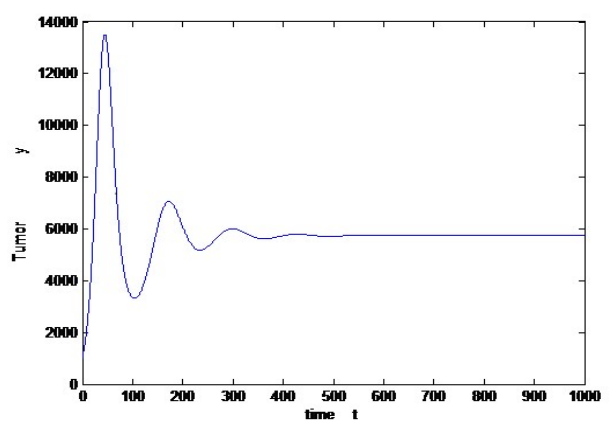

(2-b) The time response of tumor cells

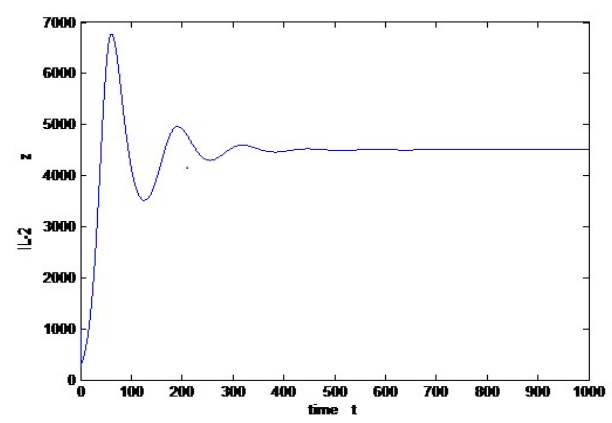

(2-c) The time response of IL-2

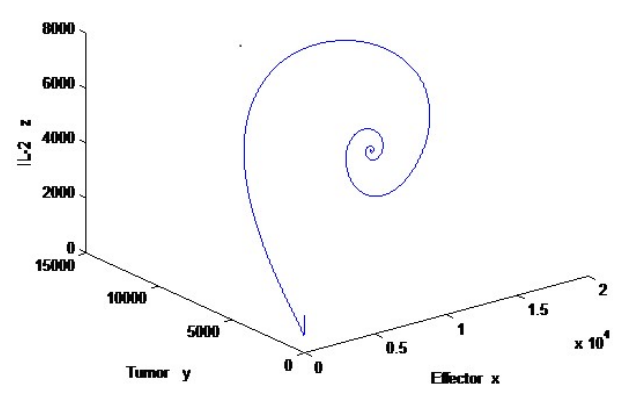

(2-d) Spiral focus of system (1)

Fig.2.The dynamical behavior and the projection of the solution of the system (1).

For the initial conditions $x_{\mathrm{o}}=200, y_{\mathrm{o}}=1 \times 10^{-7}$, and $z_{\mathrm{o}}=1 \times 10^{-7}$ and parameter values $c=.005, \mu_{2}=.03, p_{1}=.02, g_{1}=2 \times 10^{7}$,

$r_{2}=.1, b=1 \times 10^{-9}, \alpha=1, g_{2}=1 \times 10^{7}, p_{2}=5, g_{3}=1000, \mu_{3}=10$,

$s_{1}=30$, and $s_{2}=20$ the conditions $H_{1}$, and $H_{2}$ hold.

Moreover, Fig. 3 Shows that the system (1) has a unique positive periodic solution which is globally asymptotically stable. 


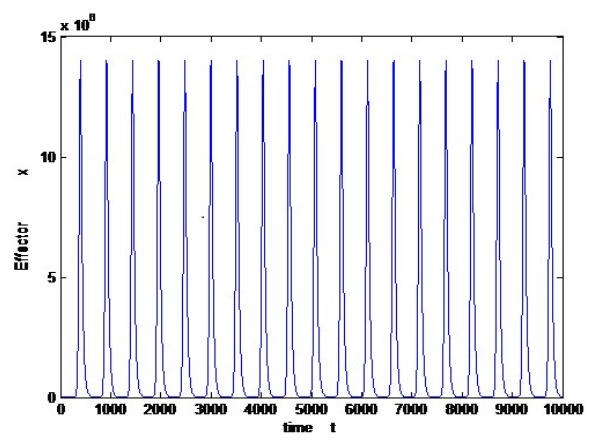

(3-a) The time response of effector cell

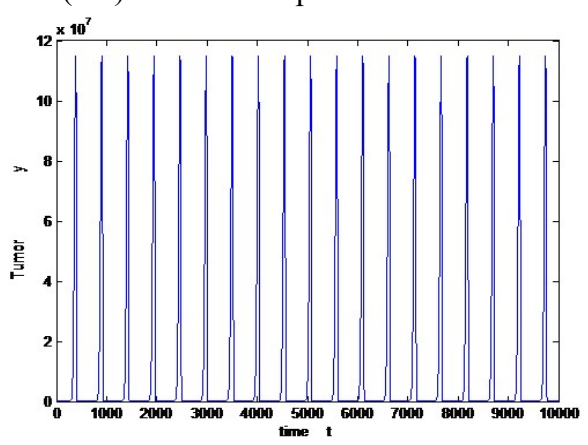

(3-b) The time response of tumor cells

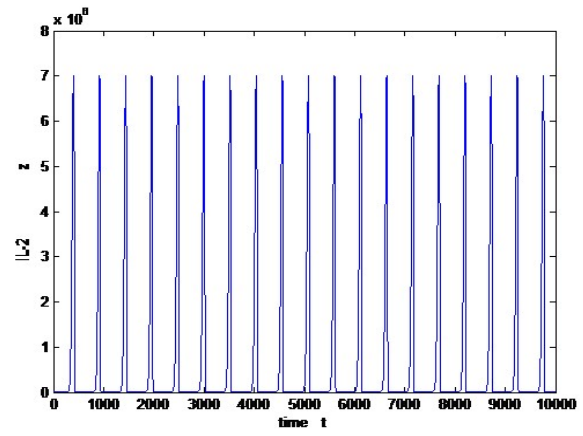

(3-c) The time response of IL-2

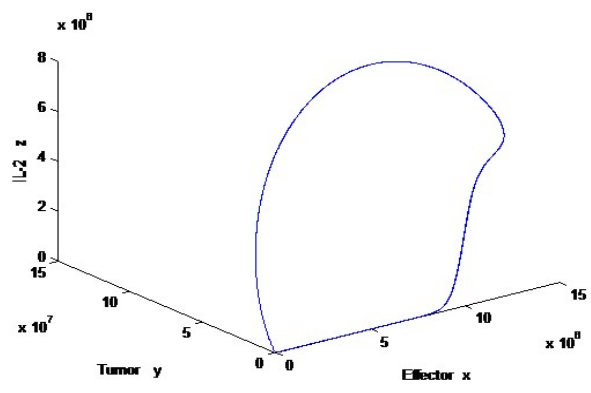

(3-d) Limit cyclic of the system (1)

Fig.3.The dynamical behavior and the projection of the solution of the system (1).

Fig.4. represents the chaotic attractor of system (1) at the the initial conditions $x_{\circ}=1000, y_{\circ}=1000$, and $z_{\circ}=1000$. with the parameter values $c=.01, \mu_{2}=.03, p_{1}=.02$, $g_{1}=2 \times 10^{7}, r_{2}=.1, b=1 \times 10^{-9}, \alpha=1, g_{2}=1 \times 10^{6}, p_{2}=5$, $g_{3}=1000, \mu_{3}=10, s_{1}=30$, and $s_{2}=20$.

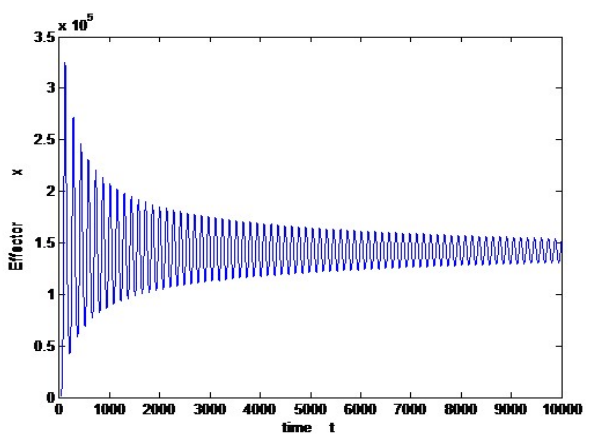

(4-a) The time response of effector cells

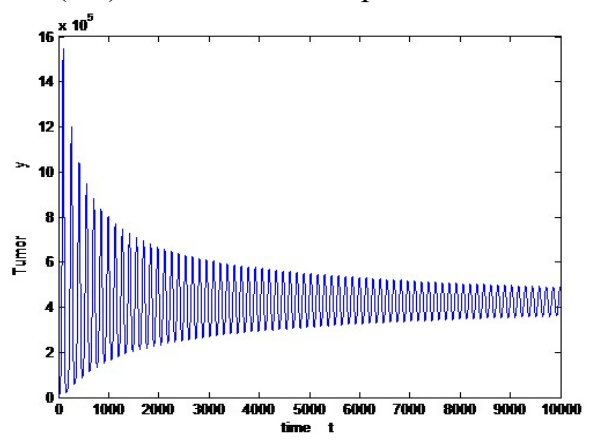

(4-b) The time response of tumor cells

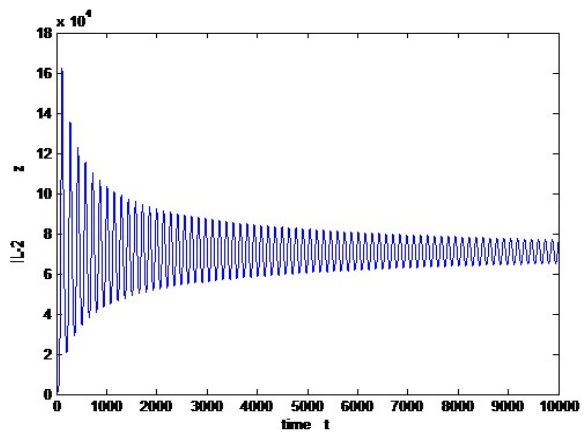

(4-c) The time response of IL-2

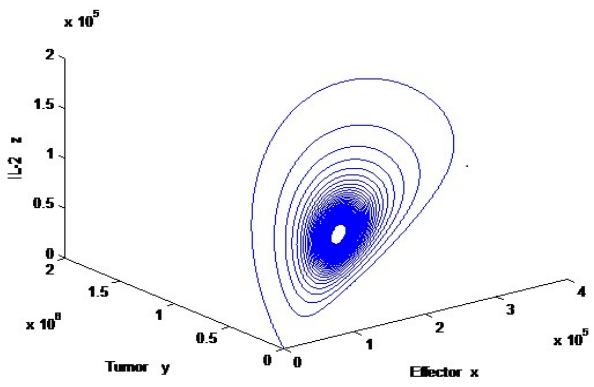

(4-d) Chaotic attractor of the system (1) Fig.4. The dynamical behavior and the projection of the solution of the system (1)

\section{7-Conclusions}

In this paper, we discuss a tumor-immune dynamical Kirschner model . We improve some results in literature. We focus on the case of immunotherapy with ACI and 
IL-Z. We established the local asymptotic stability of the tumor-free equilibrium point $E_{1}$. Our results are consistent with those obtained by Denise Kirschner et al. [7]. We prove the existence of Hopf-Andronov-Poincare bifurcation using the technique of Pimbley [11], ElSheikh.[2], and [3]. Also, we used a technique similar to that used by Changjin $\mathrm{Xu}$, and Qiing Zhang [15] to obtain sufficient conditions for the permanence of the system. By constructing a suitable Liapunov function, we give sufficient conditions guarantee the system has a unique asymptotically periodic solution which is globally asymptotically stable, see Fig.3.

\section{8-References}

[1] JA.Adam, and N.Bellomo, A Survey of models for tumor-immune system dynamics, Birkhauser Series on Modeling and Simulation in Science, Engineering and Technology, Birkhauser, Boston, MA USA1997.

[2] M.M.A.El-Sheikh, and S.A.A.El-Marouf, On stability and bifurcation of Solutions of an SEIR epidemic model with vertical transmission, International $J$. of Mathematical sciences 56(2004)2971-2987.

[3] M.M.A.El-Sheikh, and S.A.A.El-Marouf, Stability and bifurcaton of a simple food chain in a chemostat with removable rates, J. Chaos, Solitons and Fractals 23(4)(2005)1475-1489.

[4] M.M.A.El-Sheikh, S.A.A.El-Marouf and Z.M.Alaofy, On the dynamics of a general predator-prey System, Journal of Mathematics and Statistics 7(4)(2011),295-301.

[5] H.El-Owaidy, M.M.A.El-Sheikh, On stability and bifurcation of solutions of the BelousouvZhabotinskii Reaction system, J. Mathematical Analysis and Applications 151,No2(1990),438-452.

[6] Tapan Kumar Kar, Modelling and analysis of a harvested prey-predator system incorporating a prey refuge. J. Computational and Applied Mathematics 185(2006),19-33.

[7] Denise Kirschner, and John Carl Panetta, Modeling immunotherapy of tumor-immune interaction, J. Math. Biol, 37(1998),235-252.
[8] Denise Kirschner, and Alexi Tsygvigtsev, On the global dynamics of a model for tumor immunotherapy, J. Mathematical Biosciences and Engineering,6(2009),573-583.

[9] V.Kuznetsov, I.Makalkyn, M.Taylor, and A..Perelson, Nonlinear dynamics of immunogenic tumors: parameter estimation and global bifurcation analysis, Bulletin of Mathematical Biology, 56(1994)295-321.

[10] JA.Moscow, and KH.Cowan, Biology of cancer. In: Glodman L, Ausiello D.eds., Cecil Medicine. 23rd ed. Philadelphia, Pa: Saunders Elsevier; 2007:chap187.

[11] H. George Pimbley, Jr, Periodic solutions of third order predator-prey equations simulating an immune response, Arch rat Mech. Anal, 55(1974),93-123.

[12] MJ. Thun, Biology of cancer. In: Glodman L, Ausiello D.eds., Cecil Medicine. 23rd ed. Philadelphia, Pa: Saunders Elsevier; 2007:chap185.

[13] Alexei Tsygvintsev, Simeone Marino, and Denise E. Kirschner, A Mathematical model of gene therapy for the treatment of cancer, Mathematical Models and Methods in Biomedicine, Springer-verlag, Berlin, (2012)355373.

[14] Ranjit Kumar Upadhyay, and Sharada Nandan Raw, Complex dynamics of a three species foodchain model with Holling type iv functional response. J. Nonlinear Analysis: Modelling and Control, 16(2011),353-374.

[15] Changjin Xu, and Qiing Zhang ,Permanceand asymptotically periodic solution for a cyclic predator-prey model with Sigmoidalty pefunction response, Wseas Transctionson System,13(2014),668-678.

[16] R.Yuan, Existence of almost periodic solutions of functional differential equations of neutral type, J. Math. Anal. Appl.,165(1992),524-538.

[17] A.Zaghout, A.Ammar, and M.M.A.El-Sheikh, Oscillation and global attractivity in delay differential equations of population dynamics, J.appl. Comput. 77(2-3)(1996)pp195-204, 25072511. 


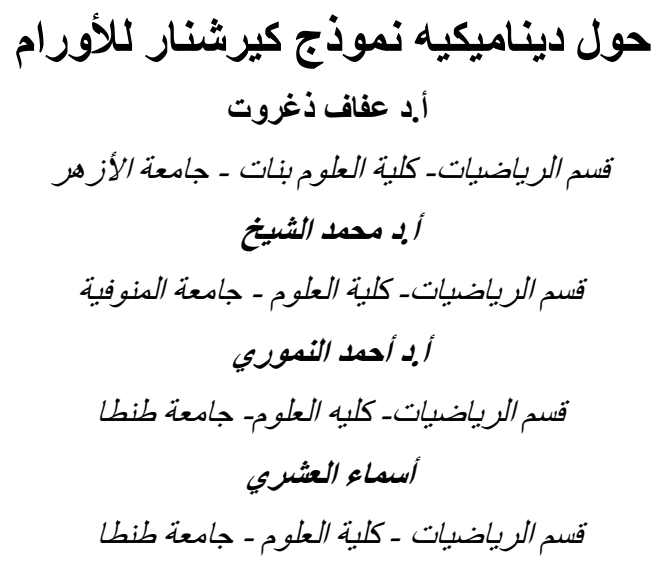

لازال السرطان يعتبر إحدى أسباب الوفاة بين البشر.ومن أسبابه المعروفة هو النمو السريع غير المحدود للخلايا أو عندما تفقد الخلايا قدرتها علي الفناء وهناك أسباب أخري للسرطان مثل التعرض للكيماويات أو الإفر اط في تناول الكحوليات أو التعرض الزائد لضوء الثمس أو اختلاف الجينات كما أن من أشهر السرطانات المؤدية للوفاة سرطان الرئة ألا أن سبب كثير من السرطانات غير معلوم حتى الآن ومن الجدير بالذكر ان نوع السرطان قد يختلف من منطقه لآخري. في 1920 قام كل من Volterra و بعمل نموذج رياضي يصف العملية السكانية وفي 1998 قام Kirschner و بتطبيق ذللك في تطوير النماذج المعرفة لنموذج السرطان وقد قاما بدر اسة سلوك الاستقر ار و التشعب لنقط الاتزان في حاله الخلو من المرض وتبع هذا البحث الكثير من الانجازات في دراسة خو اص النموذج عدديا وتحليايا في هذا البحث ندرس تحليليا نموذج Kirschner بطريقة مشابهة لنتائجنا في مساهماتنا السابقة في مجال الرياضيات الحيوية حيث ندرس في هذا البحث بعض الخواص الوصفيه لنقاط الاتزان مثل المحدودية والوجود والاستقرار والتشعب وحصلنا علي شروط كافيه لوجود حل دوري مستقر واحد وقد منا بعض التطبيقات والأمثلة العددية لتوضيح النتائج التي حصلنا عليها . 